\author{
Catherine S. C. Bouman \\ Lui G. Forni \\ Michael Joannidis
}

\title{
Biomarkers and acute kidney injury: dining with the Fisher King?
}

Received: 6 November 2009

Accepted: 7 November 2009

Published online: 3 December 2009

(C) Copyright jointly hold by Springer and ESICM 2009

This editorial refers to the articles available at: doi:10.1007/s00134-009-1711-1, 10.1007/s00134-009-1724-9 and 10.1007/s00134-009-1726-7.

\section{S. C. Bouman}

Department of Intensive Care, Academic Medical Center,

University of Amsterdam, Amsterdam, The Netherlands

L. G. Forni

Western Sussex Hospitals Trust, Brighton and Sussex Medical

School, University of Sussex, Brighton, UK

\section{Joannidis (}

Medical Intensive Care Unit, Department of Internal Medicine, Medical University of Innsbruck, Anichstrasse 35,

6020 Innsbruck, Austria

e-mail: michael.joannidis@i-med.ac.at

Tel.: +43-512-50481404

Fax: +43-512-50424199

Despite its limited utility, the serum creatinine remains an essential part of both the RIFLE and AKIN criteria for the diagnosis of acute kidney injury (AKI) [1]. Due to the delayed rise in creatinine following injury, nephrologists and intensivists alike continue to search for the holy grail of AKI: an early and reliable biomarker of kidney injury. Biomarkers are biological parameters that may indicate normal or pathological processes or responses to interventions and may be objectively quantified. The sensitivity, specificity and time course of a biomarker are critical factors in determining its use in any disease process. But more importantly, the utility of a biomarker depends on the purpose it is expected to fulfill. Summarzing current literature, it appears that an ideal biomarker for AKI should fulfill the following criteria:

- It should distinguish pre-renal AKI from apoptotic and necrotic injury

- It should be specific for renal injury in the presence of concomitant injury involving other organs

- It should allow timing of the onset or stage of injury

- It should predict outcome

- In the end it should act as surrogate end point useful for clincial interventional studies

This issue of Intensive Care Medicine includes three studies addressing the performance of two biomarkers, serum neutrophil gelatinase-associated lipocalin (NGAL) and Ang-2, addressing specific applications in AKI [2-4].

Among numerous recently published studies on biomarkers [5], NGAL has perhaps received the most attention in the literature, with 20 other studies to date supplemented by a further 2 in this edition of the journal $[6,7]$. NGAL is a ubiquitous $25-\mathrm{KDa}$ protein, covalently bound to gelatinase from human neutrophils, which is expressed at very low concentrations in various human tissues, including the kidney, trachea, lungs, stomach and colon [8]. NGAL expression increases greatly in the presence of inflammation and injured epithelia, and this includes renal damage after ischemia reperfusion injury and nephrotoxicity [9]. In a recent systematic review and meta-analysis, the NGAL meta-analysis investigator group concluded that NGAL appears to be of diagnostic and prognostic value for AKI in critically ill patients [10]. However, the role of NGAL in critically ill patients was principally studied in highly selected populations, including children and adults following cardiac surgery or contrast-induced nephropathy. Demonstration of similarly robust sensitivity and specificity in a heterogeneous ICU population would strengthen the concept of NGAL as a biomarker for AKI. The study by Cruz et al. [3] attempts 
to address this issue and assesses the diagnostic accuracy of plasma NGAL (pNGAL) both for the early detection of AKI and the need for renal replacement therapy (RRT) in an adult intensive care unit population (ICU). Blood samples were collected daily from ICU admission for up to 4 days. They used a commercially available test (Triage $^{\circledR}$ NGAL Test Biosite Inc, San Diego, CA) and in contrast to many studies prospectively examined a heterogeneous ICU population over a 7-month period. Of the 301 patients, 133 (i.e., 44\%) qualified for AKI as defined by the RIFLE creatinine and urine output criteria during ICU admission, of which roughly 50\% were in the setting of sepsis. Ninety of the 133 patients (i.e., 68\%) already had AKI at the time of first pNGAL measurement. It is unclear how many patients developed AKI within $48 \mathrm{~h}$, although this is the primary outcome of the study. Plasma NGAL was described as a fair diagnostic marker for AKI development within $48 \mathrm{~h}$ [area under ROC (AuROC) $0.78,95 \%$ CI $0.65-0.90]$, though from the manuscript it is not clear if the authors included the patients who already presented with AKI in their ROC analysis. It makes little sense to predict value in patients with established AKI, except if pNGAL predicts progression in AKI severity. The diagnostic performance of pNGAL on AKI day minus one would have been interesting, but was not reported. Interestingly, the pNGAL was found to be elevated in all ICU patients whether they had AKI or not; $67 \%$ of patients developed AKI within $24 \mathrm{~h}$ of admission, and only 37 patients progressed to a more severe RIFLE class following the development of AKI. Moreover, median pNGAL levels of patients who developed AKI within 24-48 h were not statistically significant compared to those of non-AKI patients $(p=0.13)$. What beomes evident is that pNGAL levels certainly correlate with overall disease severity as assessed by SAPS II, APACHE II and SOFA. Part of the allure associated with biomarkers is that they may help identify patients at risk of AKI. This study does not help that cause. Given that all these patients were admitted to an ICU environment, they still developed AKI despite 'best care,' and a small percentage continued to progress despite treatment.

Cruz et al. [3] did not find differences in p-NGAL between patients with sepsis and those without. This is in contrast to the findings reported by Bagshaw and colleagues in this edition of the journal [2] who examined the measurement of both plasma and urinary NGAL in 83 patients with septic and non-septic AKI. Plasma NGAL was determined using the same technique as Cruz et al. [3], whereas urinary NGAL was measured by a chemiluminescent microparticle assay using the ARCHITECT platform (Abbott Diagnostics Inc., Abbott Park, IL). Unfortunately, the authors do not specify whether they used creatinine and urine output criteria or creatinine criteria alone for determination of the RIFLE category. Also, it is not clear if the inclusion day was the first AKI day and whether the patients were enrolled at ICU admission or during their ICU treatment.

Surprisingly, no differences at enrollment between the two groups in AKI severity were demonstrated, but septic AKI was associated with significantly higher initial plasma and urine NGAL compared with non-septic AKI $(p<0.001)$. Urine NGAL remained higher in septic compared with non-septic AKI at $12(p<0.001)$ and $24 \mathrm{~h}$ $(p<0.001)$. Peak pNGAL and peak uNGAL (used for ROC estimations) showed fair diagnostic performance discriminating between septic and non-septic AKI (AuROC 0.77; 95\% CI, 0.63-0.90 and AuROC 0.70; 0.590.82 ). Once again, the 'sicker' patients demonstrated higher NGAL levels and, although not quite reaching statistical significance, showed rises in more conventional markers of renal function.

In an earlier study performed in critically ill children [11], it was demonstrated that pNGAL is increased in sepsis and septic shock even in the absence of AKI. This was not unexpected regarding the fact that NGAL is released from activated neutrophils. Also, a larger trial of 451 critically ill adults demonstrated that urine NGAL, though independently associated with AKI, yielded only very moderate discrimination at $48 \mathrm{~h}$ [12]. Consequently, and in lack of a non-AKI control group, the study by Bagshaw et al. [2] does not answer the question whether NGAL (either pNGAL or uNGAL) could help to discriminate between two different pathophysiologic entities of AKI (septic versus non-septic) or simply reflects the presence or absence of sepsis in addition to AKI.

When comparing both papers, a common theme emerges. Although NGAL estimations may predict AKI occurring within $24 \mathrm{~h}$ (and maybe even within $48 \mathrm{~h}$ ), they are not truly specific in that the other multiple problems and comorbidities our critically ill patients have can also elevate NGAL. This may also explain the fact that predictive ability of NGAL for AKI was found to be far better in children (AuROC 0.930; 95\% CI 0.883-0.968) than in adults (AuROC 0.782; 95\% CI, 0.689-0.872) in a recent meta-analysis [10].

Both studies also tried to evaluate the diagnostic performance of pNGAL to predict AKI progression and the need for RRT. Bagshaw et al. [2] found a modest predictive capacity for worsening AKI (AuROC 0.71; 95\% CI 0.55-0.88) and for the need of RRT (AuROC 0.70; 95\% CI 0.58-0.82). It must be borne in mind that only a small number of patients with AKI did progress $(N=20)$ or need RRT $(N=13)$. Cruz et al. [3] demonstrated a slightly better performance for NGAL in predicting the requirement for RRT (AuROC 0.82, 95\%CI 0.70-0.95). Although using a biomarker for predicting the need for RRT appears an interesting concept, it flies in the face of current clinical practice. Indication of RRT, in whatever form, relies on acid-base parameters, volume status and electrolyte imbalance independent, to a degree, of markers of renal function such as creatinine. There is no reason 
why early detection of AKI would confer a benefit with regards to initiation of RRT.

Predicting outcome of patients requiring RRT is addressed in the paper by Kümpers and colleagues [4] using a completely different biomarker, angiopoietin-2 (Ang-2). The angiopietin/Tie 2 signaling system in critical illness appears to play a crucial role in the symptoms of MODS, although its exact function in the pathological cascade is not fully established [13]. Ang-2, a circulating antagonistic ligand of the endothelial specific Tie2 receptor, is considered to be a 'bad guy' that induces vascular leakage [13]. Previous work by Kümpers et al. [14] suggested release of Ang-2 in endotoxemia and sepsis, and hence, in the present study they examined the utility of Ang-2 to serve as an outcome specific biomarker in critically ill patients requiring RRT [4]. Once again, the biomarker concentrations rose in tandem with severity of illness, and it did prove to be an adequate predictor of outcome in this group, confirming earlier findings in critically ill patients [13]. Perhaps the most interesting finding was that the Ang-2 levels were not significantly affected by dialysis, although no indication as to the sieving coefficient was given, and thus it remained a predictor for outcome for this selected group of patients both at the beginning and after 14 days of RRT treatment. Although Ang-2 appears capable of predicting those patients who will survive RRT, it remains doubtful whether this may be used in any way to decide on when and how long RRT should be applied in our daily clinical practice.

So does the emergence of biomarkers currently truly add anything to our clinical practice? Yes, they are markers of AKI, but both studies employing NGAL show that in a significant number of our patients this state has been reached by the time they arrive. Yes, they predict the need for RRT, but this is unsurprising. Are they a troponin for the kidney? At present, we may have reached the level of LDH or even CK at best. As we all know, tests work less well in the critical care arena, and perhaps this is where we have become blinkered. Biomarkers may have a clearer role outside of the ICU in either predicting the development of AKI or indeed in predicting potential critical illness. It may be that in this arena a potential benefit may ensue. As recently suggested [15], clinical application of biomarkers would require a four-step process of qualification: exploration by in vitro experiments and preclincal studies, demonstration of association with clinical outcomes in preclinical studies, characterization by several prospective clinical studies in humans and finally surrogacy for a clinical endpoint. A major limitation in this process is the fact that creatinine, which is a functional parameter of glomerular filtration and not a clinical end point, is used as the "gold standard" for most AKI biomarker studies. But with AKI being more than just a change in glomerular function, this "gold standard" does not exhibit an AuROC of 0.99 either [16].

Consequently, the search for the Holy Grail of (critical care) nephrology, a truly specific early marker of AKI continues.

Conflicts of interest statement The authors do declare no conflict of interest.

\section{References}

1. Mehta RL, Kellum JA, Shah SV, Molitoris BA, Ronco C, Warnock DG, Levin A (2007) Acute kidney injury network: report of an initiative to improve outcomes in acute kidney injury. Crit Care 11:R31

2. Bagshaw SM, Bennett M, Haase M, Haase-Fielitz A, Egi M, Morimatsu H, D'amico G, Goldsmith D, Devarajan P, Bellomo R (2009) Plasma and urine neutrophil gelatinase-associated lipocalin (NGAL) in septic versus nonseptic acute kidney injury in critical illness. Intensive Care Med. doi:10.1007/s00134-009-1724-9

3. Cruz DN, De Cal M, Garzotto F, Perazella M, Lentini P, Corradi V, Piccini P, Ronco C (2009) Plasma neutrophil gelatinase-associated lipocalin is an early biomarker for acute kidney injury in an adult ICU population. Intensive Care Med. doi:10.1007/s00134-009-1711-1
4. Kümpers P, Hafer C, Sascha D, Hecker $\mathrm{H}$, Lukasz A, Fliser D, Haller H, Kielstein J, Faulhaber-Walter R (2009) Angiopoietin-2 in patients requiring renal replacement therapy in the ICU: relation to acute kidney injury, multiple organ dysfunction, and outcome. Intensive Care Med. doi:10.1007/s00134-009-1726-7

5. Coca SG, Yalavarthy R, Concato J, Parikh CR (2008) Biomarkers for the diagnosis and risk stratification of acute kidney injury: a systematic review. Kidney Int 73:1008-1016

6. Endre ZH, Westhuyzen J (2008) Early detection of acute kidney injury: emerging new biomarkers. Nephrology (Carlton) 13:91-98

7. Soni SS, Ronco C, Katz N, Cruz DN (2009) Early diagnosis of acute kidney injury: the promise of novel biomarkers. Blood Purif 28:165-174
8. Cowland JB, Borregaard N (1997) Molecular characterization and pattern of tissue expression of the gene for neutrophil gelatinase-associated lipocalin from humans. Genomics 45:17-23

9. Mishra J, Ma Q, Prada A, Mitsnefes M, Zahedi K, Yang J, Barasch J, Devarajan P (2003) Identification of neutrophil gelatinase-associated lipocalin as a novel early urinary biomarker for ischemic renal injury. J Am Soc Nephrol 14:2534-2543

10. Haase M, Bellomo R, Devarajan P, Schlattmann P, Haase-Fielitz A (2009) Accuracy of neutrophil gelatinaseassociated lipocalin (NGAL) in diagnosis and prognosis in acute kidney injury: a systematic review and metaanalysis. Am J Kidney Dis. doi:10.1053/j.ajkd.2009.07.020 
11. Wheeler DS, Devarajan P, Ma Q, Harmon K, Monaco M, Cvijanovich N, Wong HR (2008) Serum neutrophil gelatinase-associated lipocalin (NGAL) as a marker of acute kidney injury in critically ill children with septic shock. Crit Care Med 36:1297-1303

12. Siew ED, Ware LB, Gebretsadik T, Shintani A, Moons KG, Wickersham N, Bossert F, Ikizler TA (2009) Urine neutrophil gelatinase-associated lipocalin moderately predicts acute kidney injury in critically ill adults. J Am Soc Nephrol 20:1823-1832
13. van Meurs M, Kurniati NF, Wulfert FM, Asgeirsdottir SA, Satchell SC, de GI, Mathieson PW, Jongman RM, Kümpers P, Zijlstra JG, Heeringa P, Molema G (2009) Shock-induced stress induces loss of microvascular endothelial Tie2 in the kidney which is not associated with reduced glomerular barrier function. Am J Physiol Renal Physiol. 297:F272-F281

14. Kümpers $P$, Van MM, David S, Molema G, Bijzet J, Lukasz A, Biertz F, Haller H, Zijlstra JG (2009) Time course of angiopoietin-2 release during experimental human endotoxemia and sepsis. Crit Care. 13:R64
15. Wagner JA, Williams SA, Webster CJ (2007) Biomarkers and surrogate end points for fit-for-purpose development and regulatory evaluation of new drugs. Clin Pharmacol Ther 81:104-107

16. Waikar SS, Betensky RA, Bonventre JV (2007) Creatinine as the gold standard for kidney injury biomarker studies? Nephrol Dial Transplant 24:3263-3265 